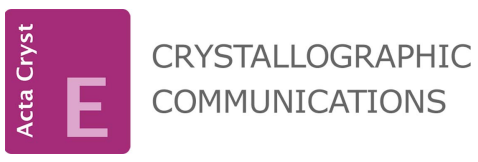

ISSN 2056-9890

Received 20 May 2015

Accepted 2 June 2015

Edited by M. Weil, Vienna University of Technology, Austria

Keywords: crystal structure; Mo clusters; reduced molybdenum selenide; monovalent indium

CCDC reference: 1404496

Supporting information: this article has supporting information at journals.iucr.org/e

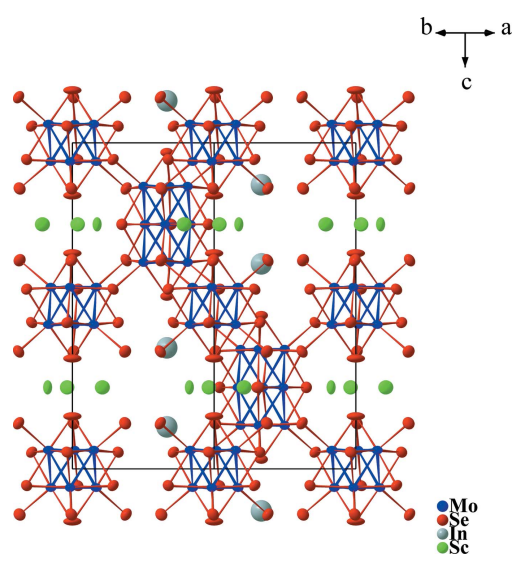

OPEN $\odot$ ACCESS

\section{Crystal structure of $\mathrm{Sc}_{1.91} \mathrm{In}_{1.39} \mathrm{Mo}_{15} \mathrm{Se}_{19 \prime}$ containing $\mathrm{Mo}_{6}$ and $\mathrm{Mo}_{9}$ clusters}

\author{
Patrick Gougeon,* Philippe Gall and Diala Salloum
}

Sciences Chimiques de Rennes, UMR CNRS No. 6226, Université de Rennes I - INSA Rennes, Avenue du Général Leclerc, 35042 Rennes CEDEX, France. *Correspondence e-mail: Patrick.Gougeon@univ-rennes1.fr

The structure of scandium indium pentadecamolybdenum nonadecaselenide, $\mathrm{Sc}_{1.91} \mathrm{In}_{1.39} \mathrm{Mo}_{15} \mathrm{Se}_{19}$, is isotypic with $\mathrm{In}_{2.9} \mathrm{Mo}_{15} \mathrm{Se}_{19}$ [Grüttner et al. (1979). Acta Cryst. B35, 285-292]. It is characterized by two cluster units $\mathrm{Mo}_{6} \mathrm{Se}_{8}^{i} \mathrm{Se}_{6}^{a}$ and $\mathrm{Mo}_{9} \mathrm{Se}_{11}^{i} \mathrm{Se}_{6}^{a}$ (where $i$ represents inner and $a$ apical atoms) that are present in a 1:1 ratio. The cluster units are centered at Wyckoff positions $2 b$ and $2 c$ and have point-group symmetry $\overline{3}$ and $\overline{6}$, respectively. The clusters are interconnected through additional $\mathrm{Mo}-\mathrm{Se}$ bonds. $\mathrm{Sc}-\mathrm{Se}$ and $\mathrm{In}-\mathrm{Se}$ bonds complete the structural set-up. In the title compound, the $\mathrm{Sc}^{3+}$ cations replace the trivalent indium atoms present in $\mathrm{In}_{2.9} \mathrm{Mo}_{15} \mathrm{Se}_{19}$, and a deficiency is observed at the monovalent indium site. One Mo, one Se and the Sc atom are situated on mirror planes, whereas two other Se atoms and the In atom are situated on threefold rotation axes.

\section{Chemical context}

From a crystal-chemical point of view, reduced molybdenum selenides $\mathrm{In}_{3} \mathrm{Mo}_{15} \mathrm{Se}_{19}$ (Grüttner et al., 1979) constitute an interesting family of compounds. Indeed, their crystal structures contain an equal mixture of $\mathrm{Mo}_{6}$ and $\mathrm{Mo}_{9}$ cluster units with the In atoms occupying two crystallographically different positions depending on their formal oxidation state of +1 or +3 . Interest in these Mo cluster compounds also lies in their physical properties because they become superconductors with high critical magnetic fields at about $4 \mathrm{~K}$ (Seeber et al., 1979). Recently, we have shown that the $\mathrm{In}^{3+}$ cation can be replaced by other trivalent cations such as $\mathrm{Ho}^{3+}$ (resulting in a compound with composition $\mathrm{Ho}_{0.76} \mathrm{In}_{1.68} \mathrm{Mo}_{15} \mathrm{Se}_{19}$; Salloum et al., 2006) or $\mathrm{V}^{3+}\left(\mathrm{V}_{1.42} \mathrm{In}_{1.83} \mathrm{Mo}_{15} \mathrm{Se}_{19}\right.$; Gougeon et al., 2010), and the $\mathrm{In}^{+}$cation by $\mathrm{K}^{+}\left(\mathrm{In}_{0.87} \mathrm{~K}_{2} \mathrm{Mo}_{15} \mathrm{Se}_{19}\right.$; Salloum et al., 2007). We present here the crystal structure of $\mathrm{Sc}_{1.91} \operatorname{In}_{1.39}$ $\mathrm{Mo}_{15} \mathrm{Se}_{19}$ in which scandium atoms replace the trivalent indium atoms.

\section{Structural commentary}

The Mo-Se framework of the title compound consists of the cluster units $\mathrm{Mo}_{6} \mathrm{Se}_{8}^{i} \mathrm{Se}_{6}^{a}$ and $\mathrm{Mo}_{9} \mathrm{Se}_{11}^{i} \mathrm{Se}_{6}{ }_{6}$ in an 1:1 ratio (for details of the $i$ - and $a$-type ligand notation, see: Schäfer $\&$ von Schnering, 1964). Both cluster units are interconnected through additional Mo-Se bonds (Table 1, Figs. 1 and 2). The first unit can be described as an $\mathrm{Mo}_{6}$ octahedron surrounded by eight face-capping inner $\mathrm{Se}^{i}$ and six apical $\mathrm{Se}^{a}$ ligands. The $\mathrm{Mo}_{9}$ cluster is surrounded by $11 \mathrm{Se}^{i}$ atoms capping one or two faces of the bioctahedron and six $\mathrm{Se}^{a}$ ligands above the apical Mo atoms. The $\mathrm{Mo}_{6} \mathrm{Se}_{8}^{i} \mathrm{Se}_{6}^{a}$ and $\mathrm{Mo}_{9} \mathrm{Se}_{11}^{i} \mathrm{Se}_{6}^{a}$ units are centered at Wyckoff positions $2 b$ and $2 c$ and have point-group 


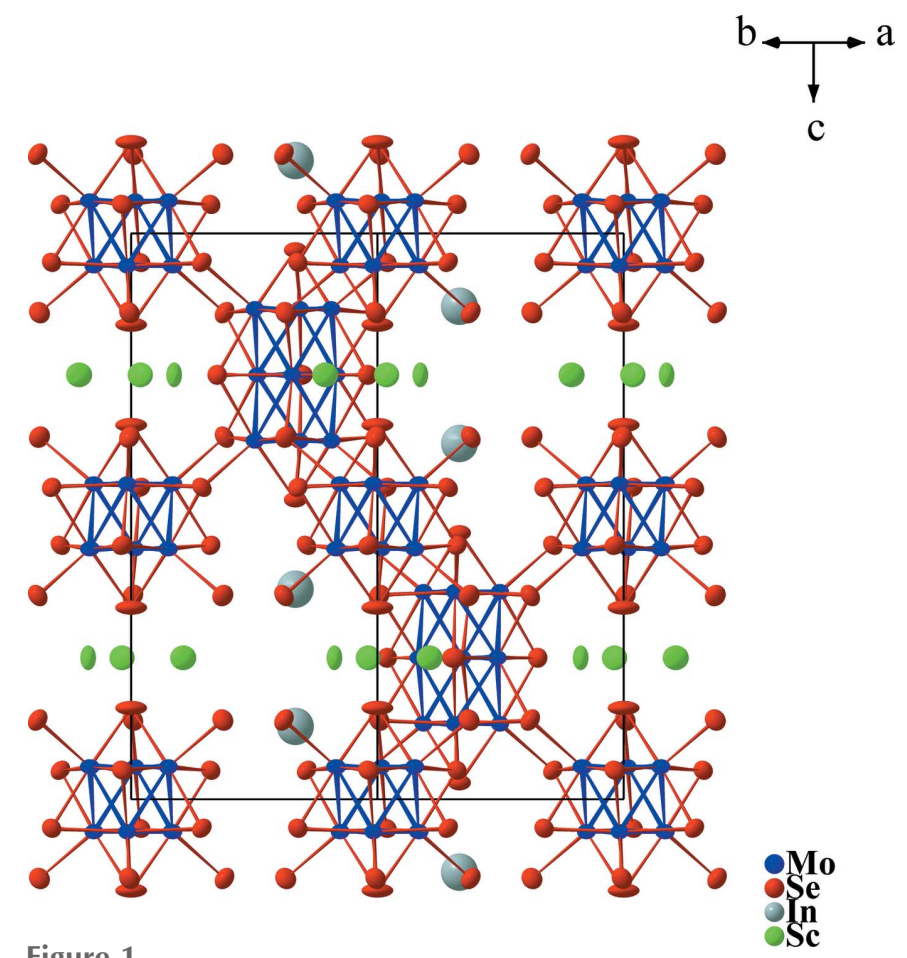

Figure 1

View of the crystal structure of $\mathrm{Sc}_{1.91} \mathrm{In}_{1.39} \mathrm{Mo}_{15} \mathrm{Se}_{19}$ along [110]. Displacement ellipsoids are drawn at the $97 \%$ probability level.

symmetry $\overline{3}$ and $\overline{6}$, respectively. The Mo-Mo distances within the $\mathrm{Mo}_{6}$ cluster are 2.6995 (6) $\AA$ for the distances of the Mo

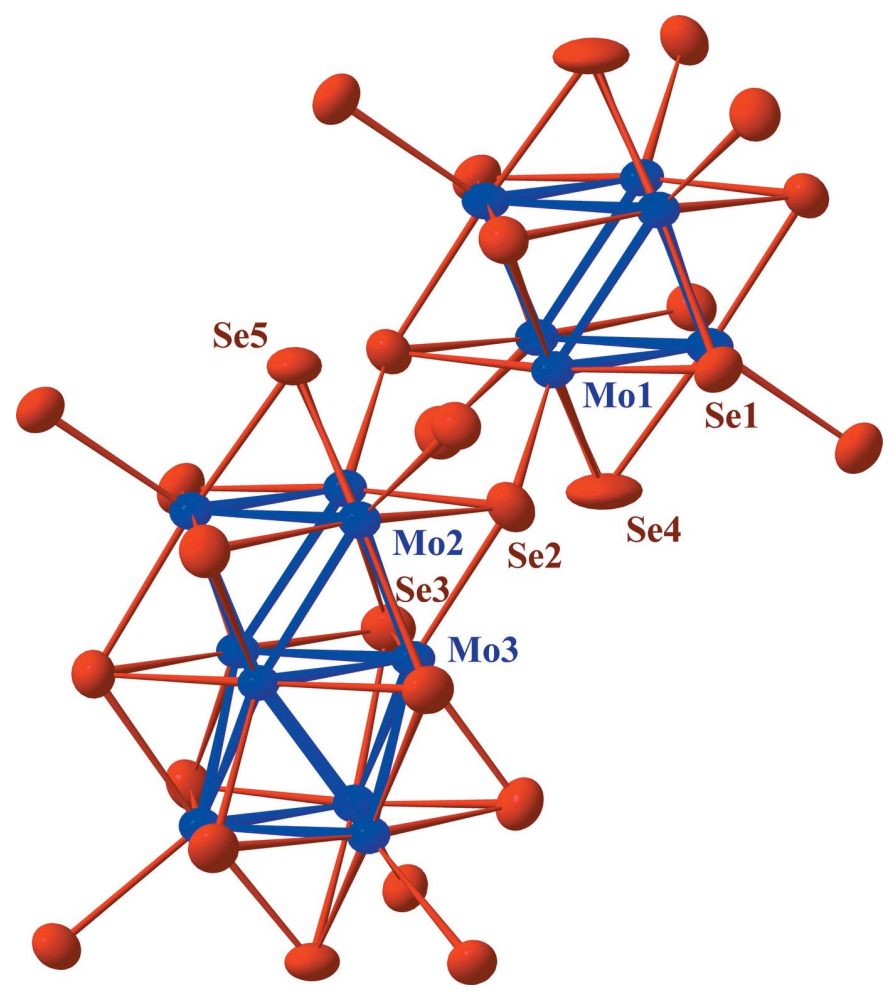

Figure 2

Plot showing the atom-numbering scheme and the interunit linkage of the $\mathrm{Mo}_{9} \mathrm{Se}_{11} \mathrm{Se}_{6}$ and $\mathrm{Mo}_{6} \mathrm{Se}_{8} \mathrm{Se}_{6}$ cluster units. Displacement ellipsoids are drawn at the $97 \%$ probability level.
Table 1

Selected bond lengths $(\AA)$.

\begin{tabular}{|c|c|c|c|}
\hline Mo1-Se4 & $2.5480(6)$ & $\mathrm{Mo} 3-\mathrm{Se} 2$ & $2.5780(5)$ \\
\hline Mo1-Se1 $1^{i}$ & $2.5488(5)$ & $\mathrm{Mo} 3-\mathrm{Se}^{\mathrm{iii}}$ & $2.5884(7)$ \\
\hline Mo1-Se1 & $2.5749(5)$ & $\mathrm{Mo} 3-\mathrm{Se} 3$ & $2.5900(7)$ \\
\hline $\mathrm{Mo} 1-\mathrm{Se} 1^{\mathrm{ii}}$ & $2.6145(5)$ & $\mathrm{Mo} 3-\mathrm{Mo}^{\mathrm{iii}}$ & $2.7127(8)$ \\
\hline Mo1-Se2 & $2.6531(5)$ & In - Se 5 & $3.0662(13)$ \\
\hline Mo1-Mo1ii & $2.6995(6)$ & $\mathrm{In}-\mathrm{Se} 2^{\mathrm{vii}}$ & $3.1273(4)$ \\
\hline Mo1-Mo1 ${ }^{\mathrm{i}}$ & 2.7179 (5) & $\mathrm{In}-\mathrm{Se} 2^{\mathrm{viii}}$ & 3.1273 (4) \\
\hline $\mathrm{Mo} 2-\mathrm{Se} 5$ & $2.5290(6)$ & $\mathrm{In}-\mathrm{Se} 2^{\mathrm{i}}$ & $3.1273(4)$ \\
\hline $\mathrm{Mo} 2-\mathrm{Se} 2$ & $2.5931(5)$ & $\mathrm{In}-\mathrm{Se} 1^{\mathrm{vii}}$ & $3.4912(6)$ \\
\hline $\mathrm{Mo} 2-\mathrm{Se} 2^{\mathrm{iii}}$ & $2.6275(5)$ & $\mathrm{In}-\mathrm{Se} 1^{\mathrm{i}}$ & 3.4912 (6) \\
\hline $\mathrm{Mo} 2-\mathrm{Mo}^{\mathrm{iv}}$ & $2.6460(6)$ & $\mathrm{In}-\mathrm{Se} 1^{\mathrm{viii}}$ & $3.4912(6)$ \\
\hline $\mathrm{Mo} 2-\mathrm{Se}^{\mathrm{v}}$ & $2.6581(5)$ & $\mathrm{Sc}-\mathrm{Se} 4^{\mathrm{vi}}$ & $2.5691(15)$ \\
\hline $\mathrm{Mo} 2-\mathrm{Se} 3^{\mathrm{iii}}$ & $2.6965(4)$ & $\mathrm{Sc}-\mathrm{Se} 3^{\mathrm{ii}}$ & $2.696(2)$ \\
\hline Mo2-Mo3 ${ }^{\mathrm{iii}}$ & $2.7196(4)$ & $\mathrm{Sc}-\mathrm{Se} 2^{\mathrm{ix}}$ & $2.8056(12)$ \\
\hline Mo2-Mo3 & 2.7675 (4) & $\mathrm{Sc}-\mathrm{Se}^{\mathrm{x}}$ & $2.8056(12)$ \\
\hline $\mathrm{Mo} 3-\mathrm{Se} 2^{\mathrm{vi}}$ & $2.5780(5)$ & $\mathrm{Sc}-\mathrm{Se} 3^{\mathrm{ix}}$ & $2.931(2)$ \\
\hline
\end{tabular}

Symmetry codes: (i) $x-y, x,-z+1$; (ii) $-y, x-y, z$; (iii) $-y+1, x-y, z$; (iv) $-x+y+1,-x+1, z ; \quad$ (v) $\quad-x+y+1,-x, z$; $\quad$ (vi) $\quad x, y,-z+\frac{3}{2}$; $\quad$ (vii) $-x+1,-y,-z+1 ; \quad$ (viii) $y+1,-x+y+1,-z+1 ; \quad$ (ix) $-x+y,-x, z ; \quad$ (x) $-x+y,-x,-z+\frac{3}{2}$.

triangles formed by the Mo1 atoms related through the threefold axis, and 2.7179(5) $\AA$ for the distances between these triangles. The $\mathrm{Mo}-\mathrm{Mo}$ distances within the $\mathrm{Mo}_{9}$ clusters are 2.6460 (6) and 2.7127 (8) $\AA$ in the triangles formed by the atoms Mo2 and Mo3, respectively, and 2.7196 (4) and 2.7675 (4) $\AA$ for those between the $\mathrm{Mo}_{3}$ and $\mathrm{Mo}_{3}$ triangles. The Se atoms bridge either one (Se1, Se2, Se4 and Se5) or two (Se3) triangular faces of the Mo clusters. Moreover, atoms Se1 and $\mathrm{Se} 2$ are linked to an Mo atom of a neighboring cluster. The Mo-Se bond lengths range from $2.5480(6)$ to 2.6531 (5) $\AA$ within the $\mathrm{Mo}_{6} \mathrm{Se}_{8}^{i} \mathrm{Se}_{6}^{a}$ unit, and from 2.5290 (6) to 2.6966 (4) $\AA$ within the $\mathrm{Mo}_{9} \mathrm{Se}_{11}^{i} \mathrm{Se}_{6}^{a}$ unit. Each $\mathrm{Mo}_{9} \mathrm{Se}_{11}^{i} \mathrm{Se}_{6}^{a}$ cluster is interconnected by six $\mathrm{Mo}_{6} \mathrm{Se}_{8}^{i} \mathrm{Se}_{6}^{a}$ units (and vice versa) via $\mathrm{Mo} 2-\mathrm{Se} 1$ bonds (and $\mathrm{Mo} 1-\mathrm{Se} 2$ bonds, respectively), forming the three-dimensional Mo-Se framework, the connectivity formula of which is $\mathrm{Mo}_{9} \mathrm{Se}_{5}^{i} \mathrm{Se}_{6 / 2}^{i-a} \mathrm{Se}_{6 / 2}^{a-i}$, $\mathrm{Mo}_{6} \mathrm{Se}_{2}^{i} \mathrm{Se}_{6 / 2}^{i-a} \mathrm{Se}_{6 / 2}^{a-i}$. It results from this arrangement that the shortest intercluster Mo1-Mo2 distance is 3.4361 (5) $\AA$, indicating only weak metal $\cdots$ metal interactions.

Comparison of the Mo-Mo and $\mathrm{Mo}-\mathrm{Se}$ distances with those of the other substituted compounds $\mathrm{Ho}_{0.76} \mathrm{In}_{1.68}$ $\mathrm{Mo}_{15} \mathrm{Se}_{19}, \mathrm{In}_{0.87} \mathrm{~K}_{2} \mathrm{Mo}_{15} \mathrm{Se}_{19}$ and $\mathrm{V}_{1.42} \mathrm{In}_{1.83} \mathrm{Mo}_{15} \mathrm{Se}_{19}$ does not reveal great differences although the cationic charges are different in the four compounds. The $\mathrm{In}^{+}$cations are surrounded by seven Se atoms, forming a distorted tricapped tetrahedron as in $\mathrm{In}_{2.9} \mathrm{Mo}_{15} \mathrm{Se}_{19}$. The $\mathrm{Se} 5$ and $\mathrm{Se} 2$ atoms forming the tetrahedron are at 3.0662 (13) and 3.1273 (4) $\AA$ from the $\mathrm{In}^{+}$cation, and the capping $\mathrm{Se} 1$ atoms are at 3.4912 (6) $\AA$. While in $\operatorname{In}_{2.9} \mathrm{Mo}_{15} \mathrm{Se}_{19}$ the monovalent In site is fully occupied, in the title compound it is only has 69.5 (3)\% occupancy. This deficiency probably results from the higher temperature used during the crystal-growth process, which led to a loss of indium and selenium because of the high volatility of these elements at $1773 \mathrm{~K}$. The $\mathrm{Sc}^{3+}$ cations, as the $\mathrm{In}^{3+}$ cations in the $\mathrm{In}_{3} \mathrm{Mo}_{15} \mathrm{Se}_{19}$ compounds, occupy partially at $63.8(6) \%$ a triangular group of distorted octahedral cavities, which are formed by two $\mathrm{Mo}_{6} \mathrm{Se}_{8}^{i} \mathrm{Se}_{6}^{a}$ and three $\mathrm{Mo}_{9} \mathrm{Se}_{11}^{i} \mathrm{Se}_{6}^{a}$ 
Table 2

Experimental details.

\begin{tabular}{|c|c|}
\hline \multicolumn{2}{|l|}{ Crystal data } \\
\hline Chemical formula & $\mathrm{Sc}_{1,91} \mathrm{In}_{1,39} \mathrm{Mo}_{15} \mathrm{Se}_{19}$ \\
\hline$M_{\mathrm{r}}$ & 3185.04 \\
\hline Crystal system, space group & Hexagonal, $P 6_{3} / m$ \\
\hline Temperature $(\mathrm{K})$ & 293 \\
\hline$a, c(\AA)$ & $9.7530(2), 19.3977(2)$ \\
\hline$V\left(\AA^{3}\right)$ & $1597.93(7)$ \\
\hline$Z$ & 2 \\
\hline Radiation type & Mo $K \alpha$ \\
\hline$\mu\left(\mathrm{mm}^{-1}\right)$ & 28.65 \\
\hline Crystal size $(\mathrm{mm})$ & $0.06 \times 0.05 \times 0.04$ \\
\hline \multicolumn{2}{|l|}{ Data collection } \\
\hline Diffractometer & Nonius KappaCCD \\
\hline Absorption correction & $\begin{array}{l}\text { Analytical (de Meulenaar \& } \\
\text { Tompa, 1965) }\end{array}$ \\
\hline$T_{\min }, T_{\max }$ & $0.279,0.424$ \\
\hline $\begin{array}{l}\text { No. of measured, independent and } \\
\text { observed }[I>2 \sigma(I)] \text { reflections }\end{array}$ & $31413,2414,1847$ \\
\hline$R_{\text {int }}$ & 0.073 \\
\hline$(\sin \theta / \lambda)_{\max }\left(\AA^{-1}\right)$ & 0.807 \\
\hline \multicolumn{2}{|l|}{ Refinement } \\
\hline$R\left[F^{2}>2 \sigma\left(F^{2}\right)\right], w R\left(F^{2}\right), S$ & $0.031,0.063,1.08$ \\
\hline No. of reflections & 2414 \\
\hline No. of parameters & 67 \\
\hline$\Delta \rho_{\max }, \Delta \rho_{\min }\left(\mathrm{e} \AA^{-3}\right)$ & $2.22,-1.97$ \\
\hline
\end{tabular}

Computer programs: COLLECT (Nonius, 1998), EVALCCD (Duisenberg et al., 2003), SIR97 (Altomare et al., 1999), SHELXL2014/6 (Sheldrick, 2015) and DIAMOND (Bergerhoff, 1996).

units, around the threefold rotation axis. The $\mathrm{Sc}-\mathrm{Se}$ distances are in the 2.5691 (15)-2.931 (2) ^ range.

\section{Synthesis and crystallization}

Single crystals of $\mathrm{Sc}_{1.91} \mathrm{In}_{1.39} \mathrm{Mo}_{15} \mathrm{Se}_{19}$ were obtained from a mixture of $\mathrm{Sc}_{2} \mathrm{Se}_{3}, \mathrm{MoSe}_{2}$, InSe and Mo with a nominal composition $\mathrm{Sc}_{2} \mathrm{In}_{2} \mathrm{Mo}_{15} \mathrm{Se}_{19}$. Before use, Mo powder was reduced under $\mathrm{H}_{2}$ flowing gas at $1273 \mathrm{~K}$ for ten $\mathrm{h}$ in order to eliminate any trace of oxygen. The binaries $\mathrm{Sc}_{2} \mathrm{Se}_{3}, \mathrm{MoSe}_{2}$, InSe were obtained by heating stoichiometric mixtures of the elements in sealed evacuated silica tubes for about two days. All handling of materials was performed in an argon-filled glove box. The initial mixture ( $c a 5 \mathrm{~g}$ ) was cold pressed and loaded into a molybdenum crucible, which was sealed under a low argon pressure using an arc welding system. The charge was heated at the rate of $300 \mathrm{~K} \mathrm{~h}^{-1}$ up to $1773 \mathrm{~K}$, the temperature which was held for $48 \mathrm{~h}$, then cooled at $100 \mathrm{~K} \mathrm{~h}^{-1}$ down to $1373 \mathrm{~K}$ and finally furnace cooled.

\section{Refinement}

Crystal data, data collection and structure refinement details are summarized in Table 2. The highest and lowest remaining electron densities are located 0.66 and $0.62 \AA$ from the In site, respectively. Refinement of the occupancy factors of the Sc and In atoms led to the final composition $\mathrm{Sc}_{1.914(12)} \operatorname{In}_{1.390(6)} \mathrm{Mo}_{15} \mathrm{Se}_{19}$.

\section{Acknowledgements}

Intensity data were collected on the Nonius KappaCCD X-ray diffactometer system of the Centre de diffractométrie de l'Université de Rennes I (URL: www.cdifx.univ-rennes1.fr).

\section{References}

Altomare, A., Burla, M. C., Camalli, M., Cascarano, G. L., Giacovazzo, C., Guagliardi, A., Moliterni, A. G. G., Polidori, G. \& Spagna, R. (1999). J. Appl. Cryst. 32, 115-119.

Bergerhoff, G. (1996). DIAMOND. University of Bonn, Germany.

Duisenberg, A. J. M., Kroon-Batenburg, L. M. J. \& Schreurs, A. M. M. (2003). J. Appl. Cryst. 36, 220-229.

Gougeon, P., Gall, P., Salloum, D. \& Potel, M. (2010). Acta Cryst. E66, i73.

Grüttner, A., Yvon, K., Chevrel, R., Potel, M., Sergent, M. \& Seeber, B. (1979). Acta Cryst. B35, 285-292.

Meulenaer, J. de \& Tompa, H. (1965). Acta Cryst. 19, 1014-1018.

Nonius (1998). COLLECT. Nonius BV, Delft, The Netherlands.

Salloum, D., Gougeon, P. \& Potel, M. (2006). Acta Cryst. E62, i83-i85.

Salloum, D., Gougeon, P. \& Potel, M. (2007). Acta Cryst. E63, i8-i10.

Schäfer, H. \& von Schnering, H. G. (1964). Angew. Chem. 76, 833849.

Seeber, B., Decroux, M., Fischer, Ø., Chevrel, R., Sergent, M. \& Grüttner, A. (1979). Solid State Commun. 29, 419-423.

Sheldrick, G. M. (2015). Acta Cryst. C71, 3-8. 


\section{supporting information}

Acta Cryst. (2015). E71, 760-762 [doi:10.1107/S2056989015010634]

\section{Crystal structure of $\mathrm{Sc}_{1.91} \ln _{1.39} \mathrm{Mo}_{15} \mathrm{Se}_{19}$, containing $\mathrm{Mo}_{6}$ and $\mathrm{Mog}_{9}$ clusters}

\section{Patrick Gougeon, Philippe Gall and Diala Salloum}

\section{Computing details}

Data collection: COLLECT (Nonius, 1998); cell refinement: COLLECT (Nonius, 1998); data reduction: EVALCCD (Duisenberg et al., 2003); program(s) used to solve structure: SIR97 (Altomare et al., 1999); program(s) used to refine structure: SHELXL2014/6 (Sheldrick, 2015); molecular graphics: DIAMOND (Bergerhoff, 1996); software used to prepare material for publication: SHELXL2014/6 (Sheldrick, 2015).

\section{Scandium indium pentadecamolybdenum nonadecaselenide}

Crystal data

$\mathrm{Sc}_{1.91} \mathrm{In}_{1.39} \mathrm{Mo}_{15} \mathrm{Se}_{19}$

$M_{r}=3185.04$

Hexagonal, $P 6_{3} / m$

$a=9.7530(2) \AA$

$c=19.3977(2) \AA$

$V=1597.93(7) \AA^{3}$

$Z=2$

\section{Data collection}

Nonius KappaCCD diffractometer

Radiation source: fine-focus sealed tube Graphite monochromator $\varphi$ scans $(\kappa=0)+$ additional $\omega$ scans Absorption correction: analytical

(de Meulenaar \& Tompa, 1965)

$T_{\min }=0.279, T_{\max }=0.424$

Refinement

Refinement on $F^{2}$

Least-squares matrix: full

$R\left[F^{2}>2 \sigma\left(F^{2}\right)\right]=0.031$

$w R\left(F^{2}\right)=0.063$

$S=1.08$

2414 reflections

67 parameters

0 restraints
$F(000)=2769$

$D_{\mathrm{x}}=6.620 \mathrm{Mg} \mathrm{m}^{-3}$

Mo $K \alpha$ radiation, $\lambda=0.71069 \AA$

$\mu=28.65 \mathrm{~mm}^{-1}$

$T=293 \mathrm{~K}$

Irregular block, black

$0.06 \times 0.05 \times 0.04 \mathrm{~mm}$

31413 measured reflections 2414 independent reflections 1847 reflections with $I>2 \sigma(I)$

$R_{\text {int }}=0.073$

$\theta_{\max }=35.0^{\circ}, \theta_{\min }=2.4^{\circ}$

$h=-13 \rightarrow 15$

$k=-15 \rightarrow 13$

$l=-31 \rightarrow 26$

$w=1 /\left[\sigma^{2}\left(F_{\mathrm{o}}^{2}\right)+(0.0228 P)^{2}+7.5263 P\right]$ where $P=\left(F_{\mathrm{o}}^{2}+2 F_{\mathrm{c}}^{2}\right) / 3$

$(\Delta / \sigma)_{\max }=0.002$

$\Delta \rho_{\max }=2.22 \mathrm{e} \AA^{-3}$

$\Delta \rho_{\min }=-1.96$ e $\AA^{-3}$

Extinction correction: SHELXL, $\mathrm{Fc}^{*}=\mathrm{kFc}\left[1+0.001 \mathrm{xFc}^{2} \lambda^{3} / \sin (2 \theta)\right]^{-1 / 4}$

Extinction coefficient: 0.00065 (4) 
Special details

Geometry. All esds (except the esd in the dihedral angle between two 1.s. planes) are estimated using the full covariance matrix. The cell esds are taken into account individually in the estimation of esds in distances, angles and torsion angles; correlations between esds in cell parameters are only used when they are defined by crystal symmetry. An approximate (isotropic) treatment of cell esds is used for estimating esds involving l.s. planes.

Fractional atomic coordinates and isotropic or equivalent isotropic displacement parameters $\left(\AA^{2}\right)$

\begin{tabular}{llllll}
\hline & $x$ & $y$ & $z$ & $U_{\text {iso }} * / U_{\text {eq }}$ & Occ. $(<1)$ \\
\hline Mo1 & $0.16732(4)$ & $0.01628(4)$ & $0.55740(2)$ & $0.00813(7)$ & \\
Mo2 & $0.68442(4)$ & $0.18633(4)$ & $0.63321(2)$ & $0.00769(7)$ & \\
Mo3 & $0.51261(5)$ & $0.16694(5)$ & 0.7500 & $0.00748(8)$ & \\
Se1 & $0.03573(5)$ & $-0.28735(5)$ & $0.55124(2)$ & $0.01007(8)$ & \\
Se2 & $0.37937(5)$ & $0.00705(5)$ & $0.64001(2)$ & $0.01114(9)$ & \\
Se3 & $0.34923(7)$ & $0.30997(7)$ & 0.7500 & $0.01107(11)$ & \\
Se4 & 0.0000 & 0.0000 & $0.66131(3)$ & $0.01933(16)$ & \\
Se5 & 0.6667 & 0.3333 & $0.52931(3)$ & $0.01116(13)$ & $0.695(3)$ \\
In & 0.6667 & 0.3333 & $0.37124(6)$ & $0.0358(4)$ & $0.638(6)$ \\
Sc & $-0.2115(2)$ & $-0.1745(2)$ & 0.7500 & $0.0143(5)$ & \\
\hline
\end{tabular}

Atomic displacement parameters $\left(\AA^{2}\right)$

\begin{tabular}{lllllll}
\hline & $U^{11}$ & $U^{22}$ & $U^{33}$ & $U^{12}$ & $U^{13}$ & $U^{23}$ \\
\hline Mo1 & $0.01005(15)$ & $0.00828(15)$ & $0.00598(13)$ & $0.00453(12)$ & $0.00026(9)$ & $0.00006(9)$ \\
Mo2 & $0.00846(14)$ & $0.00829(14)$ & $0.00626(12)$ & $0.00415(12)$ & $-0.00015(9)$ & $-0.00030(9)$ \\
Mo3 & $0.0087(2)$ & $0.0085(2)$ & $0.00563(16)$ & $0.00458(16)$ & 0.000 & 0.000 \\
Se1 & $0.01139(18)$ & $0.00980(18)$ & $0.00960(15)$ & $0.00572(15)$ & $0.00112(12)$ & $0.00197(12)$ \\
Se2 & $0.00992(18)$ & $0.01101(19)$ & $0.01187(16)$ & $0.00477(14)$ & $-0.00272(12)$ & $-0.00153(12)$ \\
Se3 & $0.0098(3)$ & $0.0142(3)$ & $0.0104(2)$ & $0.0069(2)$ & 0.000 & 0.000 \\
Se4 & $0.0264(3)$ & $0.0264(3)$ & $0.0053(3)$ & $0.01318(13)$ & 0.000 & 0.000 \\
Se5 & $0.0136(2)$ & $0.0136(2)$ & $0.0063(2)$ & $0.00679(10)$ & 0.000 & 0.000 \\
In & $0.0355(5)$ & $0.0355(5)$ & $0.0363(6)$ & $0.0178(2)$ & 0.000 & 0.000 \\
Sc & $0.0176(10)$ & $0.0064(8)$ & $0.0159(8)$ & $0.0038(7)$ & 0.000 & 0.000 \\
\hline
\end{tabular}

Geometric parameters $(\AA, \stackrel{o}{)}$

\begin{tabular}{|c|c|c|c|}
\hline Mo1—Se4 & $2.5480(6)$ & $\mathrm{Se} 3-\mathrm{Sc}^{\mathrm{ii}}$ & $2.931(2)$ \\
\hline Mo1-Se1 ${ }^{\mathrm{i}}$ & $2.5488(5)$ & $\mathrm{Se} 4-\mathrm{Mo1}^{\mathrm{iii}}$ & $2.5479(6)$ \\
\hline Mo1-Se1 & $2.5749(5)$ & $\mathrm{Se} 4-\mathrm{Mo}^{\mathrm{ii}}$ & $2.5479(6)$ \\
\hline Mo1-Se1ii & $2.6145(5)$ & $\mathrm{Se} 4-\mathrm{Sc}^{\mathrm{iii}}$ & $2.5691(15)$ \\
\hline $\mathrm{Mo} 1-\mathrm{Se} 2$ & $2.6531(5)$ & $\mathrm{Se} 4-\mathrm{Sc}^{\mathrm{ii}}$ & $2.5691(15)$ \\
\hline Mo1-Mo1ii & $2.6995(6)$ & $\mathrm{Se} 4-\mathrm{Sc}$ & $2.5691(15)$ \\
\hline Mo1-Mo1 ${ }^{\mathrm{iii}}$ & $2.6995(6)$ & $\mathrm{Se} 5-\mathrm{Mo}^{\mathrm{v}}$ & $2.5290(6)$ \\
\hline $\mathrm{Mo} 1-\mathrm{Mo}^{\mathrm{i}}$ & $2.7179(5)$ & $\mathrm{Se} 5-\mathrm{Mo} 2^{\mathrm{vi}}$ & $2.5290(6)$ \\
\hline Mo1-Mo1 $1^{\text {iv }}$ & $2.7179(5)$ & $\mathrm{In}-\mathrm{Se} 5$ & $3.0662(13)$ \\
\hline Mo2-Se5 & $2.5290(6)$ & $\mathrm{In}-\mathrm{Se} 2^{\mathrm{xi}}$ & $3.1273(4)$ \\
\hline $\mathrm{Mo} 2-\mathrm{Se} 2$ & $2.5931(5)$ & $\mathrm{In}-\mathrm{Se} 2^{\mathrm{xii}}$ & $3.1273(4)$ \\
\hline $\mathrm{Mo} 2-\mathrm{Se} 2^{\mathrm{v}}$ & $2.6275(5)$ & $\mathrm{In}-\mathrm{Se} 2^{\mathrm{i}}$ & $3.1273(4)$ \\
\hline
\end{tabular}




\begin{tabular}{|c|c|c|c|}
\hline $\mathrm{Mo} 2-\mathrm{Mo}^{\mathrm{vi}}$ & $2.6460(6)$ & $\mathrm{In}-\mathrm{Se} 1^{\mathrm{xi}}$ & $3.4912(6)$ \\
\hline $\mathrm{Mo} 2-\mathrm{Mo}^{\mathrm{v}}$ & $2.6460(6)$ & $\mathrm{In}-\mathrm{Se} 1^{\mathrm{i}}$ & $3.4912(6)$ \\
\hline $\mathrm{Mo} 2-\mathrm{Se} 1^{\mathrm{vii}}$ & $2.6581(5)$ & $\mathrm{In}-\mathrm{Se} 1^{\mathrm{xii}}$ & $3.4912(6)$ \\
\hline $\mathrm{Mo} 2-\mathrm{Se}^{\mathrm{v}}$ & $2.6965(4)$ & $\mathrm{In}-\mathrm{Se} 3^{\mathrm{iv}}$ & $4.2657(8)$ \\
\hline Mo2-Mo3 ${ }^{v}$ & $2.7196(4)$ & In-Se3 ${ }^{\text {iii }}$ & $4.2657(8)$ \\
\hline Mo2-Mo3 & $2.7675(4)$ & In $-\mathrm{Se} 3^{\text {xiv }}$ & $4.2657(8)$ \\
\hline Mo3-Se2 viii & $2.5780(5)$ & $\mathrm{In}-\mathrm{Sc}^{\mathrm{xv}}$ & $4.5565(18)$ \\
\hline Mo3-Se2 & $2.5780(5)$ & $\mathrm{In}-\mathrm{Sc}^{\mathrm{xvi}}$ & $4.5565(18)$ \\
\hline $\mathrm{Mo} 3-\mathrm{Se}^{\mathrm{v}}$ & $2.5884(7)$ & $\mathrm{In}-\mathrm{Sc}^{\mathrm{xvii}}$ & $4.5565(18)$ \\
\hline Mo3-Se3 & $2.5900(7)$ & $\mathrm{Sc}-\mathrm{Se} 4^{\mathrm{viii}}$ & $2.5691(15)$ \\
\hline Mo3-Mo3 ${ }^{v}$ & $2.7127(8)$ & $\mathrm{Sc}-\mathrm{Se} 3^{\mathrm{ii}}$ & $2.696(2)$ \\
\hline Mo3-Mo3 $3^{\text {vi }}$ & $2.7127(8)$ & $\mathrm{Sc}-\mathrm{Se} 2^{\mathrm{iii}}$ & $2.8056(12)$ \\
\hline Mo3-Mo2 $2^{\text {ix }}$ & $2.7196(4)$ & $\mathrm{Sc}-\mathrm{Se} 2^{\mathrm{xviii}}$ & $2.8056(12)$ \\
\hline $\mathrm{Mo3}-\mathrm{Mo} 2^{\mathrm{vi}}$ & $2.7196(4)$ & $\mathrm{Sc}-\mathrm{Mo} 3^{\mathrm{iii}}$ & $2.8760(18)$ \\
\hline Mo3-Mo2 $2^{\text {viii }}$ & $2.7675(4)$ & $\mathrm{Sc}-\mathrm{Se} 3^{\mathrm{iii}}$ & $2.931(2)$ \\
\hline $\mathrm{Mo} 3-\mathrm{Sc}^{\mathrm{ii}}$ & $2.8760(18)$ & $\mathrm{Sc}-\mathrm{Sc}^{\mathrm{iii}}$ & $3.305(3)$ \\
\hline Se1-Mo1 $1^{\text {iv }}$ & $2.5488(5)$ & $\mathrm{Sc}-\mathrm{Sc}^{\mathrm{ii}}$ & $3.305(3)$ \\
\hline $\operatorname{Se} 1-\mathrm{Mo} 1^{\mathrm{iii}}$ & $2.6145(5)$ & $\mathrm{Sc}-\mathrm{Mo} 1^{\mathrm{xviii}}$ & $3.7775(4)$ \\
\hline $\mathrm{Se} 1-\mathrm{Mo}^{\mathrm{x}}$ & $2.6581(5)$ & $\mathrm{Sc}-\mathrm{Mo} 1^{\mathrm{iii}}$ & $3.7775(4)$ \\
\hline $\mathrm{Se} 2-\mathrm{Mo} 2^{\mathrm{vi}}$ & $2.6275(5)$ & $\mathrm{Sc}-\mathrm{Mo} 2^{\mathrm{x}}$ & $4.0216(15)$ \\
\hline $\mathrm{Se} 2-\mathrm{Sc}^{\mathrm{ii}}$ & $2.8056(12)$ & $\mathrm{Sc}-\mathrm{Mo} 2^{\mathrm{xix}}$ & $4.0216(15)$ \\
\hline $\mathrm{Se} 3-\mathrm{Mo}^{\mathrm{vi}}$ & $2.5884(7)$ & $\mathrm{Sc}-\mathrm{Se} 1^{\text {xviii }}$ & $4.3993(10)$ \\
\hline $\mathrm{Se} 3-\mathrm{Mo} 2^{\mathrm{ix}}$ & $2.6966(4)$ & $\mathrm{Sc}-\mathrm{Se} 1^{\mathrm{iii}}$ & $4.3993(10)$ \\
\hline $\mathrm{Se} 3-\mathrm{Mo} 2^{\mathrm{vi}}$ & $2.6966(4)$ & $\mathrm{Sc}-\operatorname{In}^{\mathrm{xvi}}$ & $4.5564(18)$ \\
\hline $\mathrm{Se} 3-\mathrm{Sc}^{\mathrm{iii}}$ & $2.696(2)$ & & \\
\hline $\mathrm{Se} 4-\mathrm{Mo} 1-\mathrm{Se}^{\mathrm{i}}$ & $176.406(19)$ & Mo2 $2^{\text {ix }}-$ Mo3 - Mo2 & $145.97(2)$ \\
\hline Se4-Mo1-Se1 & $91.698(13)$ & $\mathrm{Mo} 2^{\mathrm{vi}}-\mathrm{Mo} 3-\mathrm{Mo} 2$ & $57.653(13)$ \\
\hline 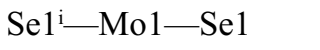 & $89.030(13)$ & $\mathrm{Se} 2^{\text {viii }}-\mathrm{Mo} 3-\mathrm{Mo}^{\text {viii }}$ & $57.907(12)$ \\
\hline $\mathrm{Se} 4-\mathrm{Mo} 1-\mathrm{Se} 1^{\mathrm{ii}}$ & $90.788(13)$ & $\mathrm{Se} 2-\mathrm{Mo} 3-\mathrm{Mo}^{\text {viii }}$ & $145.46(2)$ \\
\hline $\mathrm{Se} 1^{\mathrm{i}}-\mathrm{Mo} 1-\mathrm{Se}^{\mathrm{ii}}$ & $88.161(13)$ & $\mathrm{Se}^{\mathrm{v}}-\mathrm{Mo} 3-\mathrm{Mo}^{\mathrm{viii}}$ & $60.349(12)$ \\
\hline $\mathrm{Se} 1-\mathrm{Mo} 1-\mathrm{Se}^{\mathrm{ii}}$ & $174.05(2)$ & Se3-Mo3-Mo2 viii & $118.538(13)$ \\
\hline Se4-Mo1—Se2 & $90.338(16)$ & $\mathrm{Mo}^{\mathrm{v}}-\mathrm{Mo} 3-\mathrm{Mo}^{\text {viii }}$ & $59.495(13)$ \\
\hline $\mathrm{Se} 1$ i-Mo1-Se2 & $93.220(16)$ & $\mathrm{Mo}^{\mathrm{vi}}-\mathrm{Mo} 3-\mathrm{Mo}^{\mathrm{viii}}$ & $88.794(12)$ \\
\hline $\mathrm{Se} 1-\mathrm{Mo} 1-\mathrm{Se} 2$ & $86.430(16)$ & $\mathrm{Mo} 2^{\mathrm{ix}}-\mathrm{Mo} 3-\mathrm{Mo} 2^{\mathrm{viii}}$ & $57.654(13)$ \\
\hline $\mathrm{Se} 1^{\mathrm{ii}}-\mathrm{Mo} 1-\mathrm{Se} 2$ & $98.964(16)$ & $\mathrm{Mo}^{\mathrm{vi}}-\mathrm{Mo} 3-\mathrm{Mo}^{\mathrm{viii}}$ & $145.97(2)$ \\
\hline $\mathrm{Se} 4-\mathrm{Mo} 1-\mathrm{Mol}^{\mathrm{ii}}$ & $58.012(9)$ & Mo2-Mo3-Mo2 ${ }^{\text {viii }}$ & $109.89(2)$ \\
\hline $\mathrm{Se}^{\mathrm{i}}-\mathrm{Mo1}-\mathrm{Mol}^{\mathrm{ii}}$ & $118.654(14)$ & $\mathrm{Se} 2^{\mathrm{viii}}-\mathrm{Mo} 3-\mathrm{Sc}^{\mathrm{ii}}$ & $61.629(19)$ \\
\hline $\mathrm{Se} 1-\mathrm{Mo1}-\mathrm{Mo}^{\mathrm{ii}}$ & $119.304(17)$ & $\mathrm{Se} 2-\mathrm{Mo} 3-\mathrm{Sc}^{\mathrm{ii}}$ & $61.629(19)$ \\
\hline 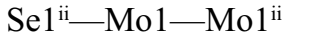 & $57.940(16)$ & $\mathrm{Se}^{\mathrm{v}}-\mathrm{Mo} 3-\mathrm{Sc}^{\mathrm{ii}}$ & $118.59(5)$ \\
\hline $\mathrm{Se} 2-\mathrm{Mo} 1-\mathrm{Mo}^{\mathrm{ii}}$ & $137.271(14)$ & $\mathrm{Se} 3-\mathrm{Mo} 3-\mathrm{Sc}^{\mathrm{ii}}$ & $64.60(4)$ \\
\hline $\mathrm{Se} 4-\mathrm{Mo} 1-\mathrm{Mo}^{\mathrm{iii}}$ & $58.012(9)$ & $\mathrm{Mo}^{\mathrm{v}}-\mathrm{Mo} 3-\mathrm{Sc}^{\mathrm{ii}}$ & $177.02(5)$ \\
\hline 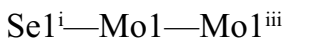 & $119.634(13)$ & $\mathrm{Mo}^{\mathrm{vi}}-\mathrm{Mo} 3-\mathrm{Sc}^{\mathrm{ii}}$ & $122.98(5)$ \\
\hline $\mathrm{Se} 1-\mathrm{Mo} 1-\mathrm{Mo}^{\mathrm{iii}}$ & $59.376(17)$ & $\mathrm{Mo} 2^{\mathrm{ix}}-\mathrm{Mo} 3-\mathrm{Sc}^{\mathrm{ii}}$ & $91.85(3)$ \\
\hline 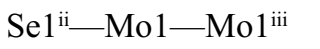 & $117.870(16)$ & $\mathrm{Mo} 2^{\mathrm{vi}}-\mathrm{Mo} 3-\mathrm{Sc}^{\mathrm{ii}}$ & $91.85(3)$ \\
\hline $\mathrm{Se} 2-\mathrm{Mo} 1-\mathrm{Mol}^{\mathrm{iii}}$ & $129.426(16)$ & $\mathrm{Mo} 2-\mathrm{Mo} 3-\mathrm{Sc}^{\mathrm{ii}}$ & $119.536(18)$ \\
\hline 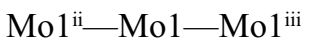 & 60.0 & 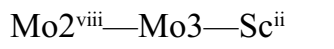 & $119.536(18)$ \\
\hline $\mathrm{Se} 4-\mathrm{Mo} 1-\mathrm{Mo}^{\mathrm{i}}$ & $118.212(12)$ & $\mathrm{Mo1}^{\mathrm{iv}}-\mathrm{Se} 1-\mathrm{Mo} 1$ & $64.071(16)$ \\
\hline
\end{tabular}




\begin{tabular}{|c|c|}
\hline $\mathrm{Se}^{\mathrm{i}}-\mathrm{Mo} 1-\mathrm{Mo}^{\mathrm{i}}$ & $58.430(15)$ \\
\hline $\mathrm{Se} 1-\mathrm{Mo1}-\mathrm{Mo}^{\mathrm{i}}$ & $117.048(15)$ \\
\hline $\mathrm{Se}^{\mathrm{ii}}-\mathrm{Mo1}-\mathrm{Mol}^{\mathrm{i}}$ & $57.068(11)$ \\
\hline $\mathrm{Se} 2-\mathrm{Mo} 1-\mathrm{Mo}^{\mathrm{i}}$ & $140.439(16)$ \\
\hline 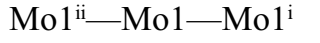 & $60.224(8)$ \\
\hline 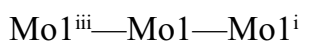 & 90.0 \\
\hline $\mathrm{Se} 4-\mathrm{Mo} 1-\mathrm{Mol}^{\mathrm{iv}}$ & $118.212(12)$ \\
\hline Se1 ${ }^{\mathrm{i}}-\mathrm{Mo} 1-\mathrm{Mo}^{\mathrm{iv}}$ & $59.423(15)$ \\
\hline Se1-Mo1-Mo1 ${ }^{\text {iv }}$ & $57.499(11)$ \\
\hline $\mathrm{Se}{ }^{\mathrm{ii}}-\mathrm{Mo} 1-\mathrm{Mol}^{\mathrm{iv}}$ & $116.606(15)$ \\
\hline $\mathrm{Se} 2-\mathrm{Mo} 1-\mathrm{Mol}^{\mathrm{iv}}$ & $132.232(15)$ \\
\hline $\mathrm{Mol}^{\mathrm{ii}}-\mathrm{Mo} 1-\mathrm{Mol}^{\mathrm{iv}}$ & 90.0 \\
\hline 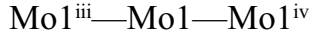 & $60.224(8)$ \\
\hline Mo1-Mo1-Mo1 ${ }^{\mathrm{iv}}$ & $59.550(15)$ \\
\hline $\mathrm{Se} 5-\mathrm{Mo} 2-\mathrm{Se} 2$ & $92.412(13)$ \\
\hline $\mathrm{Se} 5-\mathrm{Mo} 2-\mathrm{Se}^{\mathrm{v}}$ & $91.604(13)$ \\
\hline $\mathrm{Se} 2-\mathrm{Mo} 2-\mathrm{Se}_{2}^{\mathrm{v}}$ & $174.13(2)$ \\
\hline $\mathrm{Se} 5-\mathrm{Mo} 2-\mathrm{Mo} 2^{\mathrm{vi}}$ & $58.457(9)$ \\
\hline $\mathrm{Se} 2-\mathrm{Mo} 2-\mathrm{Mo} 2^{\mathrm{vi}}$ & $60.191(17)$ \\
\hline $\mathrm{Se} 2^{\mathrm{v}}-\mathrm{Mo} 2-\mathrm{Mo}^{\mathrm{vi}}$ & $118.823(16)$ \\
\hline $\mathrm{Se} 5-\mathrm{Mo} 2-\mathrm{Mo}^{\mathrm{v}}$ & $58.457(9)$ \\
\hline $\mathrm{Se} 2-\mathrm{Mo} 2-\mathrm{Mo} 2^{\mathrm{v}}$ & $120.106(17)$ \\
\hline $\mathrm{Se} 2^{\mathrm{v}}-\mathrm{Mo} 2-\mathrm{Mo}_{2}^{\mathrm{v}}$ & $58.906(16)$ \\
\hline $\mathrm{Mo} 2^{\mathrm{vi}}-\mathrm{Mo} 2-\mathrm{Mo}^{\mathrm{v}}$ & 60.0 \\
\hline $\mathrm{Se} 5-\mathrm{Mo} 2-\mathrm{Se}^{\mathrm{vii}}$ & $90.196(15)$ \\
\hline $\mathrm{Se} 2-\mathrm{Mo} 2-\mathrm{Se} 1^{\mathrm{vii}}$ & $85.764(16)$ \\
\hline $\mathrm{Se} 2^{\mathrm{v}}-\mathrm{Mo} 2-\mathrm{Se} 1^{\mathrm{vii}}$ & $98.510(16)$ \\
\hline $\mathrm{Mo} 2^{\mathrm{vi}}-\mathrm{Mo} 2-\mathrm{Se}^{\mathrm{vii}}$ & $129.579(16)$ \\
\hline $\mathrm{Mo} 2^{\mathrm{v}}-\mathrm{Mo} 2-\mathrm{Se} 1^{\mathrm{vii}}$ & $137.688(14)$ \\
\hline $\mathrm{Se} 5-\mathrm{Mo} 2-\mathrm{Se} 3^{\mathrm{v}}$ & $175.172(19)$ \\
\hline $\mathrm{Se} 2-\mathrm{Mo} 2-\mathrm{Se}^{\mathrm{v}}$ & $85.424(18)$ \\
\hline $\mathrm{Se} 2^{\mathrm{v}}-\mathrm{Mo} 2-\mathrm{Se}^{\mathrm{v}}$ & $90.244(18)$ \\
\hline $\mathrm{Mo} 2^{\mathrm{vi}}-\mathrm{Mo} 2-\mathrm{Se} 3^{\mathrm{v}}$ & $116.797(15)$ \\
\hline $\mathrm{Mo} 2^{\mathrm{v}}-\mathrm{Mo} 2-\mathrm{Se} 3^{\mathrm{v}}$ & $119.114(14)$ \\
\hline $\mathrm{Se}^{\mathrm{vii}}-\mathrm{Mo} 2-\mathrm{Se}^{\mathrm{v}}$ & $93.942(17)$ \\
\hline $\mathrm{Se} 5-\mathrm{Mo} 2-\mathrm{Mo}^{\mathrm{v}}$ & $120.527(17)$ \\
\hline $\mathrm{Se} 2-\mathrm{Mo} 2-\mathrm{Mo}^{\mathrm{v}}$ & $116.554(16)$ \\
\hline $\mathrm{Se} 2^{\mathrm{v}}-\mathrm{Mo} 2-\mathrm{Mo}^{\mathrm{v}}$ & $57.619(13)$ \\
\hline $\mathrm{Mo}^{\mathrm{vi}}-\mathrm{Mo} 2-\mathrm{Mo}^{\mathrm{v}}$ & $91.213(12)$ \\
\hline $\mathrm{Mo}^{2}-\mathrm{Mo} 2-\mathrm{Mo}^{\mathrm{v}}$ & $62.083(12)$ \\
\hline $\mathrm{Se} 1^{\mathrm{vii}}-\mathrm{Mo} 2-\mathrm{Mo}^{\mathrm{v}}$ & $138.895(18)$ \\
\hline $\mathrm{Se}^{\mathrm{v}}-\mathrm{Mo} 2-\mathrm{Mo}^{\mathrm{v}}$ & $57.133(16)$ \\
\hline $\mathrm{Se} 5-\mathrm{Mo} 2-\mathrm{Mo} 3$ & $118.709(16)$ \\
\hline Se2-Mo2-Mo3 & $57.381(13)$ \\
\hline $\mathrm{Se} 2 \mathrm{v}-\mathrm{Mo} 2-\mathrm{Mo} 3$ & $116.832(16)$ \\
\hline $\mathrm{Mo} 2^{\mathrm{vi}}-\mathrm{Mo} 2-\mathrm{Mo} 3$ & $60.264(12)$ \\
\hline $\mathrm{Mo} 2^{\mathrm{v}}-\mathrm{Mo} 2-\mathrm{Mo} 3$ & $90.162(12)$ \\
\hline $\mathrm{Se}^{\mathrm{vii}}-\mathrm{Mo} 2-\mathrm{Mo} 3$ & $131.676(18)$ \\
\hline
\end{tabular}

\begin{tabular}{|c|c|}
\hline $\mathrm{Mol}^{\mathrm{iv}}-\mathrm{Se} 1-\mathrm{Mol}^{\mathrm{iii}}$ & $63.509(16)$ \\
\hline Mo1-Se1-Mo1 ${ }^{\mathrm{iii}}$ & $62.685(17)$ \\
\hline $\mathrm{Mo} 1^{\mathrm{iv}}-\mathrm{Se} 1-\mathrm{Mo} 2^{\mathrm{x}}$ & $131.401(19)$ \\
\hline $\mathrm{Mo1}-\mathrm{Se} 1-\mathrm{Mo}^{\mathrm{x}}$ & $128.246(18)$ \\
\hline 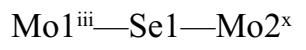 & $81.335(15)$ \\
\hline Mo3-Se2-Mo2 & $64.712(15)$ \\
\hline $\mathrm{Mo} 3-\mathrm{Se} 2-\mathrm{Mo} 2^{\mathrm{vi}}$ & $62.984(14)$ \\
\hline $\mathrm{Mo} 2-\mathrm{Se} 2-\mathrm{Mo} 2^{\mathrm{vi}}$ & $60.903(17)$ \\
\hline Mo3-Se2-Mo1 & $130.17(2)$ \\
\hline Mo2-Se2-Mo1 & $126.687(18)$ \\
\hline $\mathrm{Mo} 2^{\mathrm{vi}}-\mathrm{Se} 2-\mathrm{Mo} 1$ & $81.187(15)$ \\
\hline $\mathrm{Mo} 3-\mathrm{Se} 2-\mathrm{Sc}^{\mathrm{ii}}$ & $64.42(3)$ \\
\hline $\mathrm{Mo} 2-\mathrm{Se} 2-\mathrm{Sc}^{\mathrm{ii}}$ & $129.13(3)$ \\
\hline $\mathrm{Mo}^{\mathrm{vi}}-\mathrm{Se} 2-\mathrm{Sc}^{\mathrm{ii}}$ & $95.44(4)$ \\
\hline $\mathrm{Mo} 1-\mathrm{Se} 2-\mathrm{Sc}^{\mathrm{ii}}$ & $87.53(3)$ \\
\hline Mo3 ${ }^{\text {vi }}-\mathrm{Se} 3-\mathrm{Mo} 3$ & $63.18(2)$ \\
\hline $\mathrm{Mo} 3^{\mathrm{vi}}-\mathrm{Se} 3-\mathrm{Mo} 2^{\mathrm{ix}}$ & $63.118(13)$ \\
\hline $\mathrm{Mo} 3-\mathrm{Se} 3-\mathrm{Mo} 2^{\mathrm{ix}}$ & $61.881(13)$ \\
\hline $\mathrm{Mo}^{\mathrm{vi}}-\mathrm{Se} 3-\mathrm{Mo} 2^{\mathrm{vi}}$ & $63.118(13)$ \\
\hline $\mathrm{Mo} 3-\mathrm{Se} 3-\mathrm{Mo}^{\mathrm{vi}}$ & $61.881(14)$ \\
\hline $\mathrm{Mo} 2^{\mathrm{ix}}-\mathrm{Se} 3-\mathrm{Mo} 2^{\mathrm{vi}}$ & $114.31(2)$ \\
\hline $\mathrm{Mo}^{\mathrm{vi}}-\mathrm{Se} 3-\mathrm{Sc}^{\mathrm{iii}}$ & $162.59(5)$ \\
\hline $\mathrm{Mo} 3-\mathrm{Se} 3-\mathrm{Sc}^{\mathrm{iii}}$ & $134.23(5)$ \\
\hline $\mathrm{Mo} 2^{\mathrm{ix}}-\mathrm{Se} 3-\mathrm{Sc}^{\mathrm{iii}}$ & $121.404(15)$ \\
\hline $\mathrm{Mo}^{\mathrm{vi}}-\mathrm{Se} 3-\mathrm{Sc}^{\mathrm{iii}}$ & $121.404(15)$ \\
\hline $\mathrm{Mo}^{\mathrm{vi}}-\mathrm{Se} 3-\mathrm{Sc}^{\mathrm{ii}}$ & $125.62(4)$ \\
\hline $\mathrm{Mo} 3-\mathrm{Se} 3-\mathrm{Sc}^{\mathrm{ii}}$ & $62.43(4)$ \\
\hline $\mathrm{Mo} 2^{\mathrm{ix}}-\mathrm{Se} 3-\mathrm{Sc}^{\mathrm{ii}}$ & $91.14(2)$ \\
\hline $\mathrm{Mo} 2^{\mathrm{vi}}-\mathrm{Se} 3-\mathrm{Sc}^{\mathrm{ii}}$ & $91.14(2)$ \\
\hline $\mathrm{Sc}^{\mathrm{iii}}-\mathrm{Se} 3-\mathrm{Sc}^{\mathrm{ii}}$ & $71.79(8)$ \\
\hline 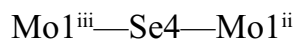 & $63.974(19)$ \\
\hline 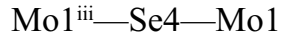 & $63.975(19)$ \\
\hline 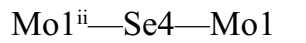 & $63.974(19)$ \\
\hline $\mathrm{Mol}^{\mathrm{iii}}-\mathrm{Se} 4-\mathrm{Sc}^{\mathrm{iii}}$ & $148.00(4)$ \\
\hline $\mathrm{Mol}^{\mathrm{ii}}-\mathrm{Se} 4-\mathrm{Sc}^{\mathrm{iii}}$ & $95.16(3)$ \\
\hline $\mathrm{Mo} 1-\mathrm{Se} 4-\mathrm{Sc}^{\mathrm{iii}}$ & $130.64(4)$ \\
\hline $\mathrm{Mo}^{\mathrm{iii}}-\mathrm{Se} 4-\mathrm{Sc}^{\mathrm{ii}}$ & $130.64(4)$ \\
\hline $\mathrm{Mo}^{\mathrm{ii}}-\mathrm{Se} 4-\mathrm{Sc}^{\mathrm{ii}}$ & $148.00(4)$ \\
\hline $\mathrm{Mo} 1-\mathrm{Se} 4-\mathrm{Sc}^{\mathrm{ii}}$ & $95.16(3)$ \\
\hline $\mathrm{Sc}^{\mathrm{iii}}-\mathrm{Se} 4-\mathrm{Sc}^{\mathrm{ii}}$ & $80.06(5)$ \\
\hline 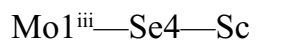 & $95.16(3)$ \\
\hline $\mathrm{Mo1}^{\mathrm{ii}}-\mathrm{Se} 4-\mathrm{Sc}$ & $130.64(4)$ \\
\hline $\mathrm{Mo} 1-\mathrm{Se} 4-\mathrm{Sc}$ & $148.00(4)$ \\
\hline $\mathrm{Sc}^{\mathrm{iii}}-\mathrm{Se} 4-\mathrm{Sc}$ & $80.06(5)$ \\
\hline $\mathrm{Sc}{ }^{\mathrm{ii}}-\mathrm{Se} 4-\mathrm{Sc}$ & $80.06(5)$ \\
\hline $\mathrm{Mo} 2^{\mathrm{v}}-\mathrm{Se} 5-\mathrm{Mo} 2$ & $63.086(18)$ \\
\hline $\mathrm{Mo} 2^{\mathrm{v}}-\mathrm{Se} 5-\mathrm{Mo} 2^{\mathrm{vi}}$ & $63.086(18)$ \\
\hline $\mathrm{Mo} 2-\mathrm{Se} 5-\mathrm{Mo} 2^{\mathrm{vi}}$ & $63.086(18)$ \\
\hline
\end{tabular}




\begin{tabular}{|c|c|}
\hline $\mathrm{Se}^{\mathrm{v}}-\mathrm{Mo} 2-\mathrm{Mo} 3$ & $56.533(15)$ \\
\hline Mo3 ${ }^{v}-\mathrm{Mo} 2-\mathrm{Mo} 3$ & $59.250(17)$ \\
\hline $\mathrm{Se} 2^{\text {viii }-M o 3}-\mathrm{Se} 2$ & $111.71(3)$ \\
\hline $\mathrm{Se} 2^{\text {viii }}-\mathrm{Mo} 3-\mathrm{Se}^{\mathrm{v}}$ & $87.997(16)$ \\
\hline $\mathrm{Se} 2-\mathrm{Mo} 3-\mathrm{Se}^{\mathrm{v}}$ & $87.997(16)$ \\
\hline 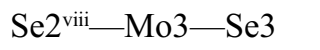 & $93.784(17)$ \\
\hline $\mathrm{Se} 2-\mathrm{Mo} 3-\mathrm{Se} 3$ & $93.784(17)$ \\
\hline $\mathrm{Se}^{\mathrm{v}}-\mathrm{Mo} 3-\mathrm{Se} 3$ & $176.82(2)$ \\
\hline $\mathrm{Se}^{\text {viii }}-\mathrm{Mo} 3-\mathrm{Mo}^{\mathrm{v}}$ & $117.324(17)$ \\
\hline $\mathrm{Se} 2-\mathrm{Mo} 3-\mathrm{Mo}^{\mathrm{v}}$ & $117.324(17)$ \\
\hline $\mathrm{Se}^{\mathrm{v}}-\mathrm{Mo} 3-\mathrm{Mo}^{\mathrm{v}}$ & $58.44(2)$ \\
\hline $\mathrm{Se} 3-\mathrm{Mo3}-\mathrm{Mo}^{\mathrm{v}}$ & $118.38(2)$ \\
\hline $\mathrm{Se}^{\mathrm{viii}}-\mathrm{Mo} 3-\mathrm{Mo}^{\text {vi }}$ & $120.613(15)$ \\
\hline $\mathrm{Se} 2-\mathrm{Mo} 3-\mathrm{Mo}^{\mathrm{vi}}$ & $120.614(15)$ \\
\hline $\mathrm{Se}^{\mathrm{v}}-\mathrm{Mo} 3-\mathrm{Mo}^{\mathrm{vi}}$ & $118.44(2)$ \\
\hline Se3-Mo3-Mo3 $3^{\text {vi }}$ & $58.38(2)$ \\
\hline $\mathrm{Mo}^{\mathrm{v}}-\mathrm{Mo} 3-\mathrm{Mo}^{\mathrm{vi}}$ & 60.0 \\
\hline 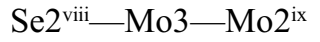 & $59.397(12)$ \\
\hline $\mathrm{Se} 2-\mathrm{Mo} 3-\mathrm{Mo}^{\mathrm{ix}}$ & $150.43(2)$ \\
\hline $\mathrm{Se} 3^{\mathrm{v}}-\mathrm{Mo} 3-\mathrm{Mo} 2^{\mathrm{ix}}$ & $118.002(13)$ \\
\hline $\mathrm{Se} 3-\mathrm{Mo} 3-\mathrm{Mo}^{\mathrm{ix}}$ & $60.986(12)$ \\
\hline $\mathrm{Mo}^{\mathrm{v}}-\mathrm{Mo} 3-\mathrm{Mo}^{\mathrm{ix}}$ & $89.795(12)$ \\
\hline $\mathrm{Mo}^{\mathrm{vi}}-\mathrm{Mo} 3-\mathrm{Mo}^{\mathrm{ix}}$ & $61.256(13)$ \\
\hline 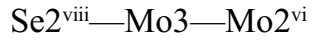 & $150.43(2)$ \\
\hline $\mathrm{Se} 2-\mathrm{Mo} 3-\mathrm{Mo}^{\mathrm{vi}}$ & $59.397(12)$ \\
\hline $\mathrm{Se} 3^{\mathrm{v}}-\mathrm{Mo} 3-\mathrm{Mo} 2^{\mathrm{vi}}$ & $118.002(13)$ \\
\hline $\mathrm{Se} 3-\mathrm{Mo} 3-\mathrm{Mo} 2^{\mathrm{vi}}$ & $60.986(12)$ \\
\hline $\mathrm{Mo}^{\mathrm{v}}-\mathrm{Mo} 3-\mathrm{Mo} 2^{\mathrm{vi}}$ & $89.795(12)$ \\
\hline $\mathrm{Mo}^{\mathrm{vi}}-\mathrm{Mo} 3-\mathrm{Mo}^{\mathrm{vi}}$ & $61.256(13)$ \\
\hline $\mathrm{Mo} 2^{\mathrm{ix}}-\mathrm{Mo} 3-\mathrm{Mo}^{\mathrm{vi}}$ & $112.82(2)$ \\
\hline $\mathrm{Se}^{\mathrm{viii}}-\mathrm{Mo} 3-\mathrm{Mo} 2$ & $145.46(2)$ \\
\hline $\mathrm{Se} 2-\mathrm{Mo} 3-\mathrm{Mo} 2$ & $57.907(12)$ \\
\hline $\mathrm{Se} 3^{\mathrm{v}}-\mathrm{Mo} 3-\mathrm{Mo} 2$ & $60.350(12)$ \\
\hline $\mathrm{Se} 3-\mathrm{Mo} 3-\mathrm{Mo} 2$ & $118.538(13)$ \\
\hline $\mathrm{Mo}^{\mathrm{v}}-\mathrm{Mo} 3-\mathrm{Mo} 2$ & $59.495(13)$ \\
\hline Mo3 $3^{\text {vi }}-\mathrm{Mo} 3-\mathrm{Mo} 2$ & $88.794(12)$ \\
\hline
\end{tabular}

\begin{tabular}{|c|c|}
\hline $\mathrm{Se} 4^{\mathrm{viii}}-\mathrm{Sc}-\mathrm{Se} 4$ & $84.08(6)$ \\
\hline $\mathrm{Se} 4^{\mathrm{viii}}-\mathrm{Sc}-\mathrm{Se} 3^{\mathrm{ii}}$ & $88.06(5)$ \\
\hline $\mathrm{Se} 4-\mathrm{Sc}-\mathrm{Se} 3^{\mathrm{ii}}$ & $88.06(5)$ \\
\hline 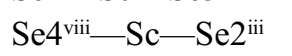 & $163.50(8)$ \\
\hline $\mathrm{Se} 4-\mathrm{Sc}-\mathrm{Se} 2^{\mathrm{iii}}$ & $86.578(17)$ \\
\hline $\mathrm{Se} 3^{\mathrm{ii}}-\mathrm{Sc}-\mathrm{Se} 2^{\mathrm{iii}}$ & $105.20(5)$ \\
\hline $\mathrm{Se} 4^{\text {viii }-\mathrm{Sc}}-\mathrm{Se} 2^{\text {xviii }}$ & $86.579(17)$ \\
\hline $\mathrm{Se} 4-\mathrm{Sc}-\mathrm{Se} 2^{\mathrm{xviii}}$ & $163.51(8)$ \\
\hline $\mathrm{Se} 3^{\mathrm{ii}}-\mathrm{Sc}-\mathrm{Se} 2^{\text {xviii }}$ & $105.20(5)$ \\
\hline $\mathrm{Se} 2^{\mathrm{iii}}-\mathrm{Sc}-\mathrm{Se} 2^{\mathrm{xviii}}$ & $99.02(6)$ \\
\hline 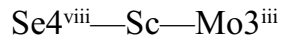 & $120.92(5)$ \\
\hline $\mathrm{Se} 4-\mathrm{Sc}-\mathrm{Mo} 3^{\mathrm{iii}}$ & $120.92(5)$ \\
\hline $\mathrm{Se} 3^{\mathrm{ii}}-\mathrm{Sc}-\mathrm{Mo} 3^{\mathrm{iii}}$ & $138.83(8)$ \\
\hline 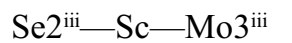 & $53.95(3)$ \\
\hline $\mathrm{Se} 2^{\text {xviii }}-\mathrm{Sc}-\mathrm{Mo} 3^{\mathrm{iii}}$ & $53.95(3)$ \\
\hline $\mathrm{Se} 4^{\mathrm{viii}}-\mathrm{Sc}-\mathrm{Se} 3^{\mathrm{iii}}$ & $83.19(5)$ \\
\hline $\mathrm{Se} 4-\mathrm{Sc}-\mathrm{Se} 3^{\mathrm{iii}}$ & $83.19(5)$ \\
\hline $\mathrm{Se} 3^{\mathrm{ii}}-\mathrm{Sc}-\mathrm{Se} 3^{\mathrm{iii}}$ & $168.21(8)$ \\
\hline 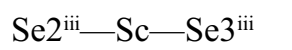 & $82.23(4)$ \\
\hline $\mathrm{Se} 2^{\mathrm{xvii}}-\mathrm{Sc}-\mathrm{Se} 3^{\mathrm{iii}}$ & $82.23(4)$ \\
\hline $\mathrm{Mo}^{\mathrm{iii}}-\mathrm{Sc}-\mathrm{Se} 3^{\mathrm{iii}}$ & $52.97(3)$ \\
\hline 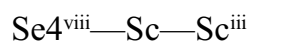 & $49.97(2)$ \\
\hline $\mathrm{Se} 4-\mathrm{Sc}-\mathrm{Sc} c^{\mathrm{iii}}$ & $49.97(2)$ \\
\hline $\mathrm{Se} 3^{3 i}-\mathrm{Sc}-\mathrm{Sc}^{\mathrm{iii}}$ & $57.39(6)$ \\
\hline $\mathrm{Se} 2^{\mathrm{iii}-\mathrm{Sc}}-\mathrm{Sc}^{\mathrm{iii}}$ & $129.92(3)$ \\
\hline $\mathrm{Se} 2^{\mathrm{xviii}}-\mathrm{Sc}-\mathrm{Sc}^{\mathrm{iii}}$ & $129.92(3)$ \\
\hline $\mathrm{Mo} 3^{\mathrm{iii}}-\mathrm{Sc}-\mathrm{Sc}^{\mathrm{iii}}$ & $163.78(9)$ \\
\hline 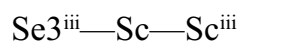 & $110.81(7)$ \\
\hline $\mathrm{Se} 4^{\mathrm{viii}-\mathrm{Sc}-\mathrm{Sc}} \mathrm{c}^{\mathrm{ii}}$ & $49.97(2)$ \\
\hline $\mathrm{Se} 4-\mathrm{Sc}-\mathrm{Sc}^{\mathrm{ii}}$ & $49.97(2)$ \\
\hline $\mathrm{Se} 3^{3 i}-\mathrm{Sc}-\mathrm{Sc}^{\mathrm{ii}}$ & $117.39(6)$ \\
\hline $\mathrm{Se} 2^{\mathrm{iii}}-\mathrm{Sc}-\mathrm{Sc}^{\mathrm{ii}}$ & $114.00(7)$ \\
\hline 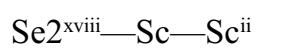 & $114.00(7)$ \\
\hline $\mathrm{Mo}^{\mathrm{iii}}-\mathrm{Sc}-\mathrm{Sc}^{\mathrm{ii}}$ & $103.78(9)$ \\
\hline 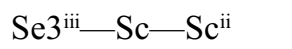 & $50.81(7)$ \\
\hline $\mathrm{Sc}^{\mathrm{iii}}-\mathrm{Sc}-\mathrm{Sc}^{\mathrm{ii}}$ & 60.0 \\
\hline
\end{tabular}

Symmetry codes: (i) $x-y, x,-z+1$; (ii) $-y, x-y, z$; (iii) $-x+y,-x, z$; (iv) $y,-x+y,-z+1$; (v) $-y+1, x-y, z$; (vi) $-x+y+1,-x+1, z$; (vii) $-x+y+1,-x, z$; (viii) $x, y$, $-z+3 / 2$; (ix) $-x+y+1,-x+1,-z+3 / 2$; (x) $-y, x-y-1, z$; (xi) $-x+1,-y,-z+1$; (xii) $y+1,-x+y+1,-z+1$; (xiii) $x-y+1, x,-z+1$; (xiv) $-x+1,-y+1,-z+1$; (xv) $y+1,-x+y,-z+1$; (xvi) $-x,-y,-z+1$; (xvii) $x-y+1, x+1,-z+1$; (xviii) $-x+y,-x,-z+3 / 2$; (xix) $-y, x-y-1,-z+3 / 2$. 